\title{
THE TREATMENT OF ADOLESCENT SLIPPING OF THE UPPER FEMORAL EPIPHYSIS *
}

\section{Denis M. Dunn, Colchester, England}

Slipped upper femoral epiphysis presents in six different ways, and a different method of treatment is required in each case.

Acute traumatic displacement; hip previously normal or nearly normal-In these cases there is a major injury to a previously normal or nearly normal hip (Fig. $1 \dagger$ ). It is obvious that something

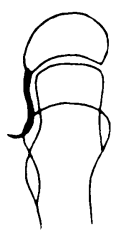

Normal

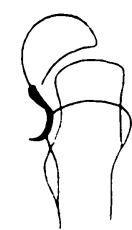

Displaced

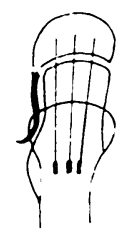

Reduced and Pinned

FiG. 1

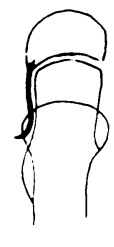

Normal

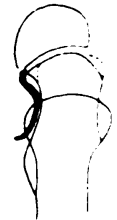

Displaced

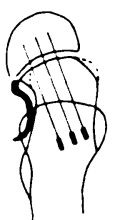

Pinned in Situ

FIG. 2

Figure 1-Acute traumatic displacement. Hip previously normal. Pinning after manipulative reduction. Figure 2-Early chronic slip. Pinning without reduction.
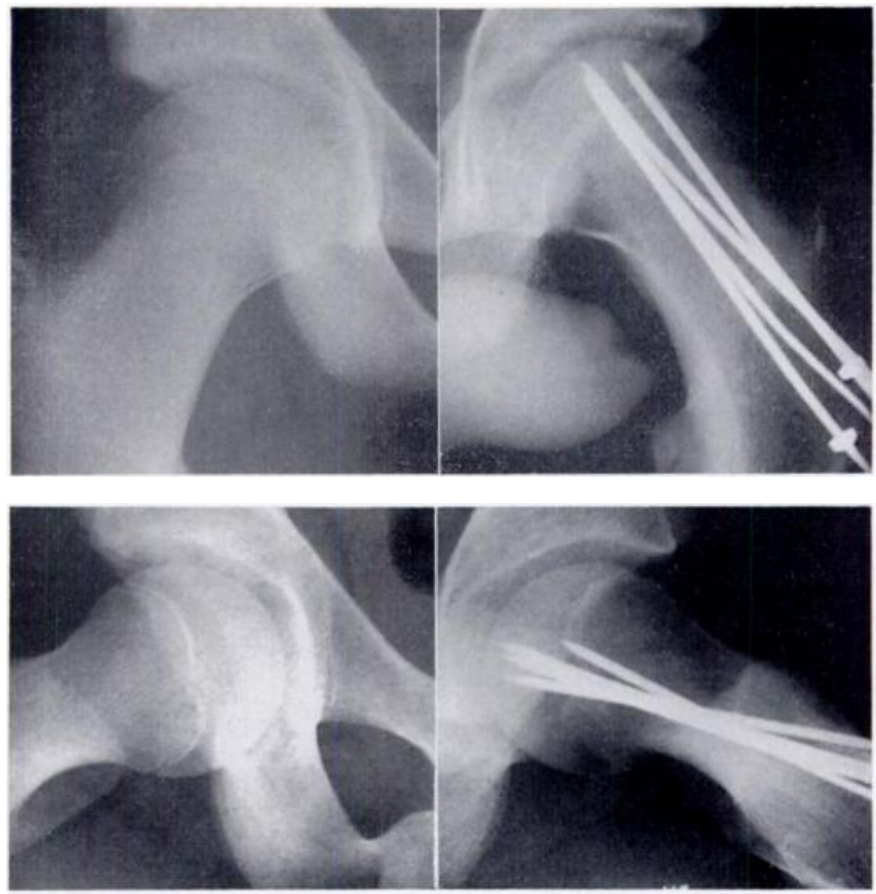

Fig. 3

Early chronic slip. Antero-posterior and (below) lateral radiographs showing early chronic slip on one side $(r i g h t)$. Pinning in position of displacement.

serious has happened and the child is admitted to hospital within a few hours. This is an uncommon injury. In the last fifteen years in the Colchester district we have seen only one undoubted case-that of a child of five who fell out of an upper window. There were also

* Based on a paper read to the British Orthopaedic Association in September 1963.

f In all the diagrams the hip is viewed from the lateral side. The retinacular vessels are indicated in each case. 
two adolescents who had probably had minor slips before the major injury. This is the only type of slipping of the upper femoral epiphysis for which closed manipulation and pinning is

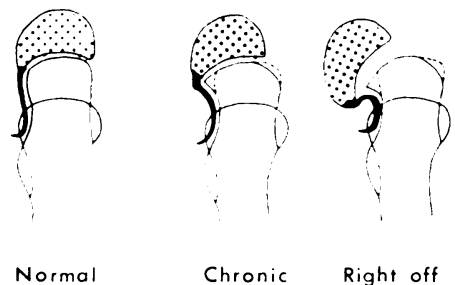

FIG. 4 permissible. The condition is an emergency; the deformity should be reduced as soon as possible-within a matter of hours-and the femoral head fixed by closed pinning (Fig. 1). Unless the patient is treated within a few hours closed manipulation should not be undertaken.

Early chronic slip-There is no problem here. If the slip is of only moderate degree the femoral head may be stabilised without manipulation by closed pinning (Figs. 2 and 3 ).

Acute on chronic slip. Note the deformity of the femoral neck.

Acute on chronic slip-A child with a slowly increasing displacement of the femoral head may have an acute exacerbation from some minor trauma such as a fall at football or from a bicycle (Fig. 4). The pain is often not severe; the child is rested at home for a few days and only comes to the clinic a week or two later because symptoms persist. There is a marked limp, but considering the looseness of the epiphysis it is remarkable that walking is possible at all.

The lateral radiograph in Figure 5 shows the displacement and also the deformity of the neck, which curves backwards as if it were trying to " catch up " with the head. This is the result of new bone which has been laid down under the periosteum stripped from the neck by the previous slow displacement. Even in the absence of previous symptoms it clearly differentiates the condition from an acute traumatic slip in a previously normal hip.

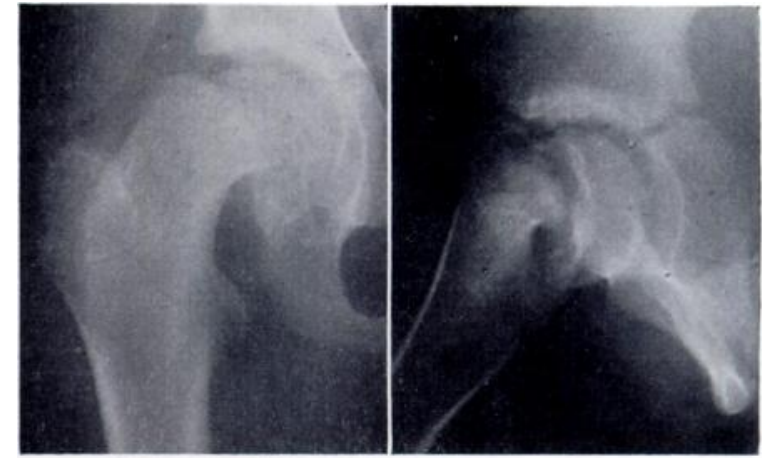

FIG. 5

Acute on chronic slip. Antero-posterior and (right) lateral radiographs showing marked displacement.

The retinacular vessels will have shortened after a few days; so no attempt should be made to effect closed reduction either by heavy traction or by manipulation, since either is likely to stretch the blood vessels (Fig. 6). These hips are best treated by open operation with shortening of the neck by trapezoid osteotomy (Fig. 7).

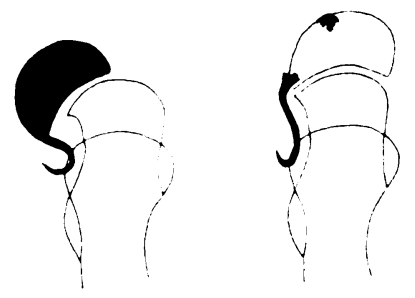

Fig. 6
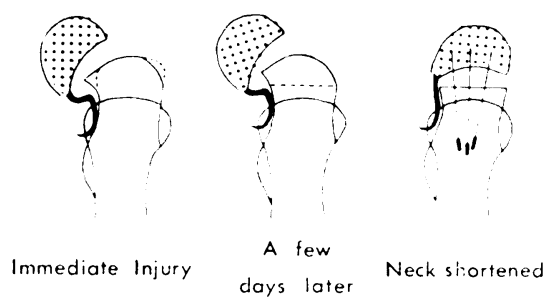

Fig. 7

Figure 6-The danger of manipulative reduction for acute on chronic slip. The shortened retinacular vessels are stretched and may be occluded when the head is replaced. Figure 7-Trapezoid osteotomy for acute on chronic slip. Note the shortening of the neck and the consequent relaxation of the retinacular vessels when the head is replaced.

Severe chronic slip, epiphysial line still open-The displacement may progress slowly without any acute symptoms to give a severe and unacceptable degree of deformity (Fig. 8), but there 
are many border-line cases in which one has to decide whether to pin the head in the position of deformity or to carry out a surgical correction either in the cervical or the trochanteric region. If the amount of slipping is less than one-third of the diameter of the femoral neck the deformity can usually be accepted; if it is more, correction should be done. Sometimes, however, secondary moulding of the neck makes it difficult to estimate the exact degree of slipping.

In deciding whether to make the osteotomy in the cervical or in the trochanteric region consideration should be given to the late results as well as to the immediate problem of the viability of the femoral head (Fig. 9). I am inclined to think that the risk of a few dead heads may be worth while if taking it will prevent the late onset of arthritis in most cases. But we must make sure that the risk is small. The hip should be entered through a lateral approach and the retinacular vessels must be protected by careful elevation of the synovium from the back of the femoral neck before replacing the head on the end of the neck.

Chronic severe slip, epiphysial line closed-Cervical osteotomy has no part to play here because vessels will be

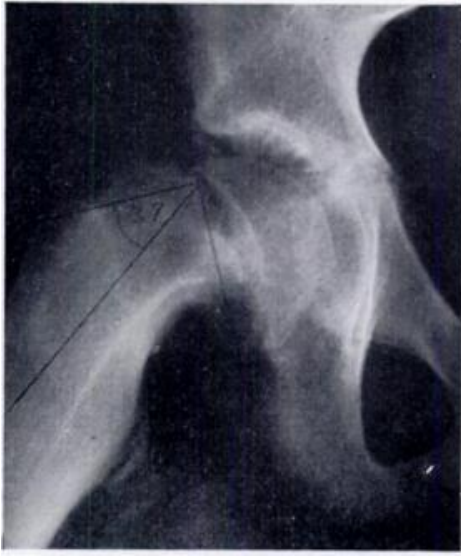

FIG. 8

Radiograph showing severe chronic slip. Epiphysial line open. growing up the neck into the head and would be destroyed by the operation (Fig. 10). The deformity of the leg can be masked by trochanteric osteotomy, and if the stub of the neck impinges on the rim of the acetabulum it can be excised.

Secondary arthritis in early adult life-This is the condition that one hopes to prevent by open correction of the original deformity (Fig. 11).

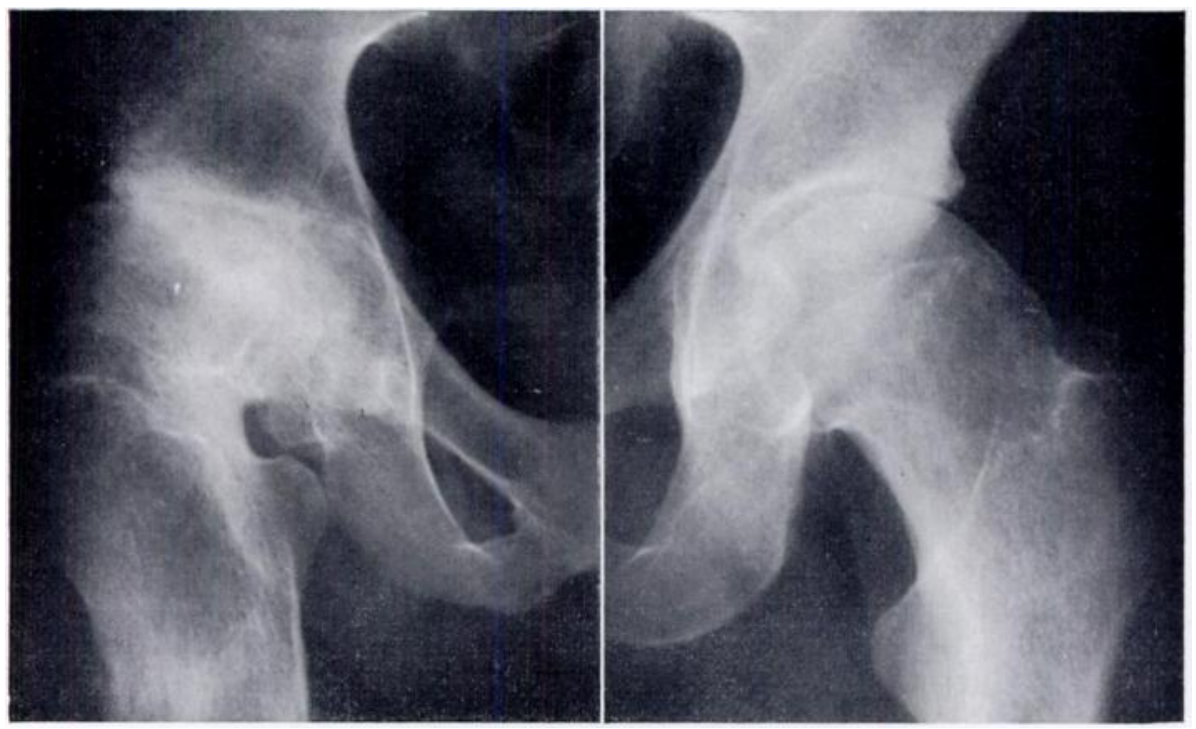

FIG. 9

Radiograph showing a late result of osteotomy. Twenty-two years after osteotomy of the right femur (left) there is severe degeneration of the hip. The left hip was treated by closed methods.

\section{TECHNIQUE OF CERVICAL OSTEOTOMY}

Exposure of the hip from the side by turning the gluteus maximus backwards, dividing the base of the greater trochanter and turning it up with the gluteus medius and minimus

VOL. 46 B, NO. 4, NOVEMBER 1964 
affords a much better view than does an anterior approach, and it is convenient for placing the pins. If the trochanter is left in place (McFarland and Osborne 1954) the view is not so good. The capsule is opened by a T-shaped incision running round the edge of the acetabulum and down the lateral aspect of the joint to the base of the trochanter. Inside the hip the most striking feature is the contrast between the smooth dull white covering of the front of

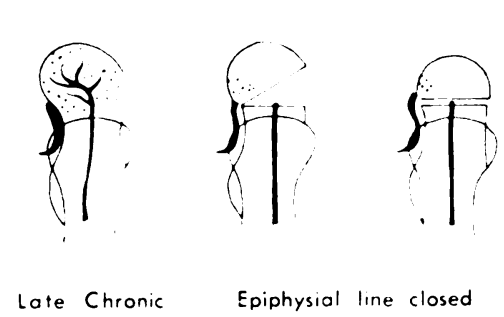

Fig. 10

The danger of cervical osteotomy for severe chronic slip with closed epiphysial line. The division of the bone destroys the vessels in the femoral neck. the neck and the highly vascular red velvety membrane at the back (Fig. 12). The appearance is quite different from that in the adult, but can be observed in operations for open reduction of congenital dislocation in young children. The end of the neck from which the head has been displaced is covered with white fibrocartilage and it may be difficult to tell where this graduates into the articular cartilage of the head.

If an acute injury has followed a chronic slip the vascular membrane is torn off the back of the neck but is still attached to the posterior rim of the head. The proximal end of the neck is bare (Fig. 13). If the acute displacement has been present for more than a day or two the retinacular vessels will have shortened and any manipulation which improves the position of the head on the neck must stretch them and may cause necrosis (Fig. 6).

The primary object of the operation is to replace the femoral head on the end of the neck without stretching the retinacular vessels. This can be achieved in one of two ways: 1) The neck can be shortened by a cut just distal to the epiphysial line and at right angles to the long axis of the neck, so as to include about a quarter of an inch of the posterior aspect of the neck (Fig. 7). The segment of bone removed is trapezoidal rather than cuneiform and includes the posterior bony beak. This procedure is suitable for those cases in which the epiphysis has been loosened by a recent injury. 2) If accidental trauma can detach the head and tear the synovium from the back of the neck without pulling the vessels out of the head, the surgeon should be able to do so as well. With care this can be done and it is the method applicable to cases of chronic severe slip without a recent acute episode. The synovial membrane is incised up the antero-lateral aspect of the neck in front of the vascular area to the edge of the head and then round the anterior margin of the head. Working slowly and gently a little at a time, the head is eased off the neck with a gouge and the synovium is raised from the back of the neck right down to the base where it turns to line the inside of the capsule. The back of the neck can now be seen and the bony beak can be

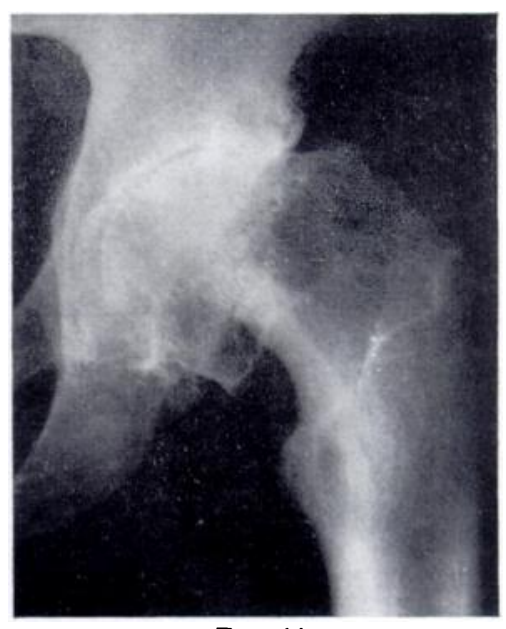

FiG. 11

Radiograph showing degeneration of hip after untreated epiphysiolysis. cut off and the stump trimmed level. It is then possible for the head to be replaced, the whole posterior synovium moving up the neck free of tension (Fig. 14).

Care after operation consists of a month on light skin traction. Hip movements are started as soon as the immediate discomfort has settled. The child is up on crutches at the end of the first month, by which time the hip usually flexes to above a right angle. Partial weight bearing is started as soon as there is radiological evidence of commencing union of the epiphysis-usually at about three months. A few weeks after this the child goes on to unrestricted activity, and the pins are removed a year or so later. 


\section{MATERIAL}

There were thirty-one patients-twenty-one boys and ten girls-ten of whom had both hips affected. Seventeen right hips and twenty-four left hips wcre affected (Table I).

Eighteen hips were subjected to closed pinning, and twenty-three to open reduction. Of the ten patients with bilateral affection six had one hip treated by closed pinning and the other

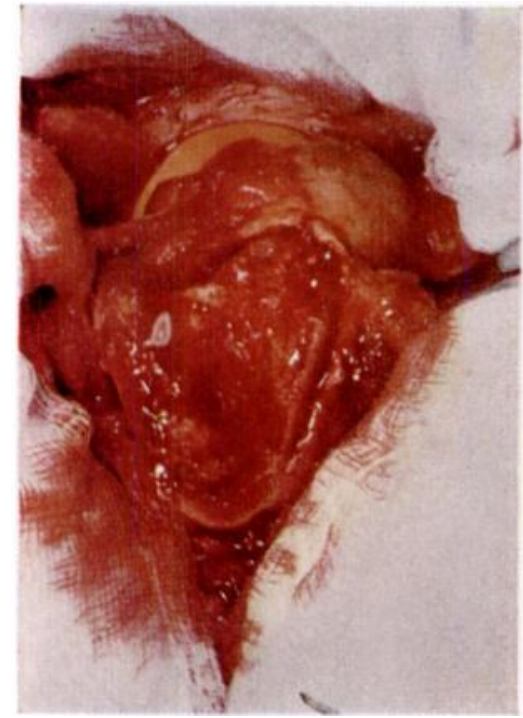

Fig. 12

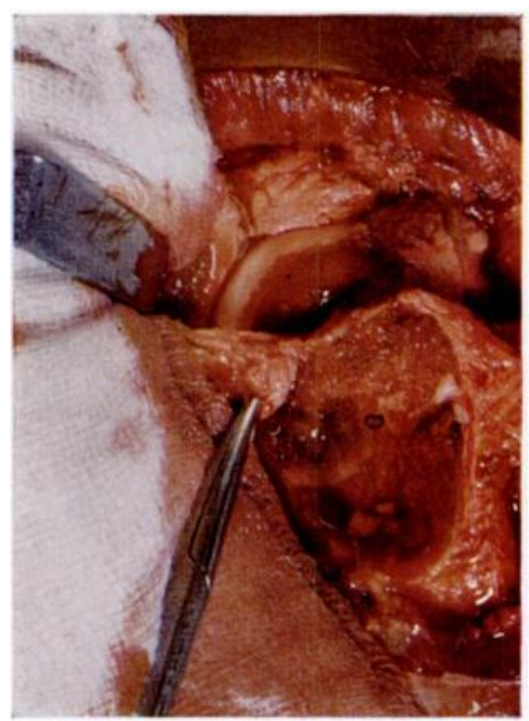

FIG. 13

Findings at operation. Figure 12-Severe chronic slip. Epiphysial line open. Note the contrast between the smooth white covering of the front of the neck and the vascular membrane covering the back (left). Figure 13-Acute on chronic slip. The vascular membrane has been torn off the back of the neck but is still attached to the posterior rim of the head.

by open reduction, three had both treated by closed pinning, and one had open reduction on both hips (Table II).

The duration of follow-up ranged from over twelve years (three patients) to six months, the average duration being six and a half years.

\section{RESULTS}

Closed pinning-Seventeen hips did very well, becoming clinically quite normal and suffering no complication. In one case a subtrochanteric fracture followed the removal of Moore's pins. They are hard to remove, and trifin nails are hard to introduce, so I now use Crawford Adams' pins. Open reduction-Nineteen hips did very well and went on to become clinically quite normal. Some patients who had open reduction on one side and closed pinning on the other
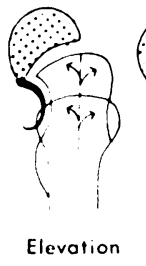

$$
\text { of Synovium }
$$
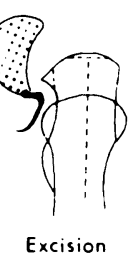

of Beak

Fig. 14

Advancement of posterior synovium to preserve its vessels.

now have a better range of movement in the hip subjected to open reduction (Fig. 15). Four hips developed complications.

Segmental necrosis of femoral head - In one case an area of necrosis developed in relation to the tip of a trifin nail. The nail itself may have been the cause of the necrosis. Nine years later the patient-a girl-has what seems to her a normal hip. There is in fact some limitation of abduction (Figs. 16 and 17).

Alascular necrosis-This occurred in one case. The radiograph before operation showed a gross degree of slip, and possibly relative density of the femoral head (Fig. 18). Six weeks 

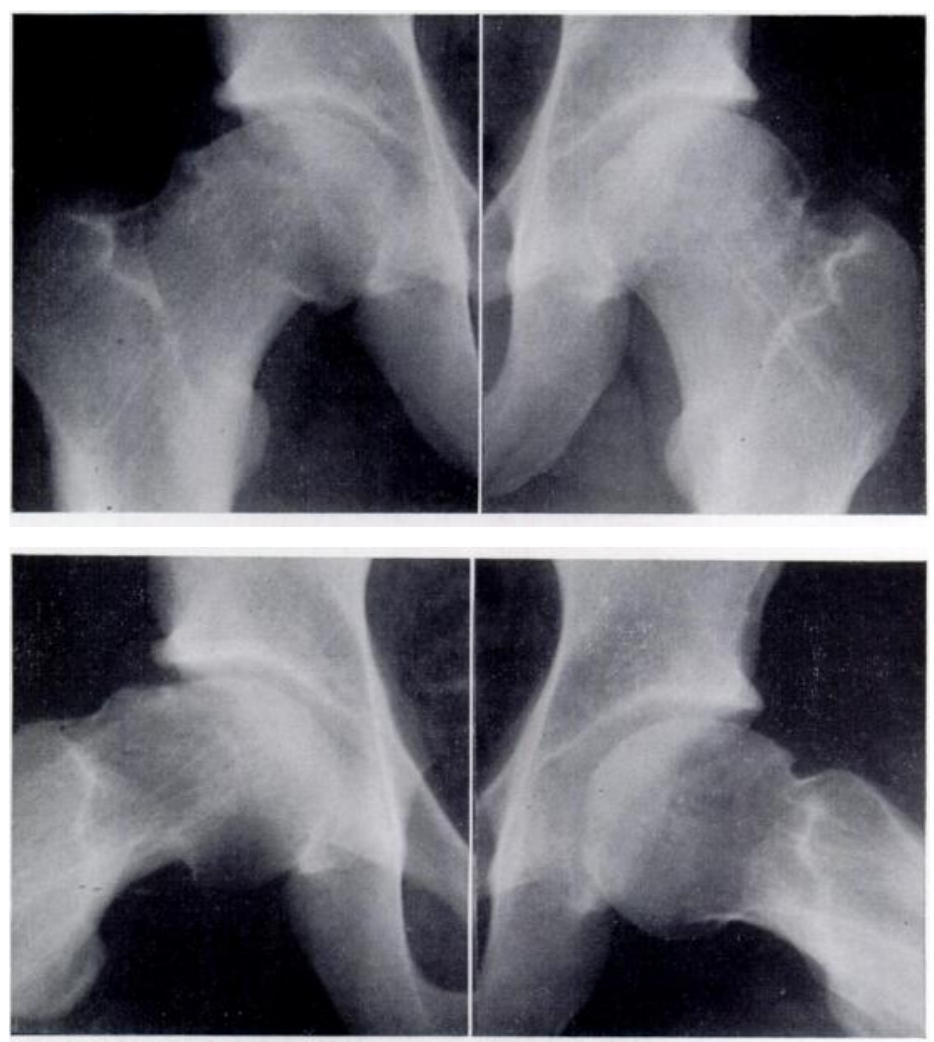

FIG. 15

Comparison of results of open reduction and closed pinning. Anteroposterior and (below) lateral radiographs four years after open reduction for the left hip (right) and closed pinning for the right hip (left). The deformity of the right femoral head is noticeable, whereas the left closely approaches the normal state.

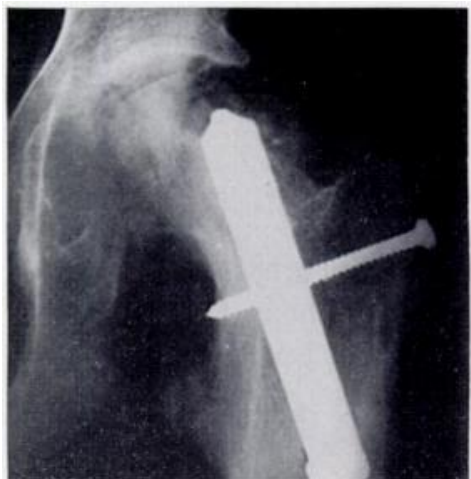

Fig. 16

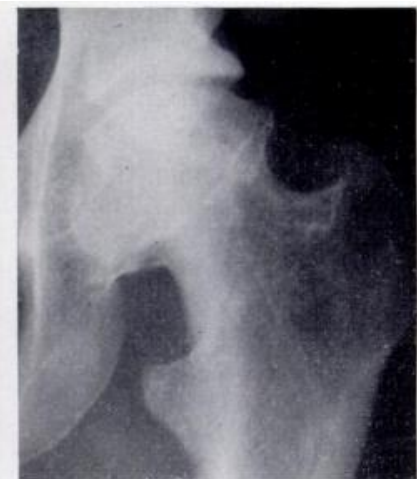

Fig. 17

Segmental necrosis at tip of nail. Figure 16-Radiograph taken some months after operation, showing necrosis in relation to the tip of the nail.

Figure 17-Nine years later: some deformity of head of femur. 
after operation the head was certainly dead (Fig. 19). The hip was arthrodesed and a good result was secured.

Acute necrosis of cartilage-This occurred in two cases. In the first case open reduction was done by McFarland's approach. Because the head and neck were poorly displayed the bone was in error divided through the middle of the neck instead of at the epiphysis. Radiographs

TABLE I

Clinical Material

\begin{tabular}{|c|c|}
\hline Total patients & 31 \\
\hline Boys & 21 \\
\hline Girls & 10 \\
\hline Total hips & 41 \\
\hline Right. & 17 \\
\hline Left & 24 \\
\hline Bilateral & 10 \\
\hline
\end{tabular}

TABLE II

Operations Performed

\begin{tabular}{|c|c|}
\hline Nature of operation & Number of hips \\
\hline Closed pinning & 18 \\
\hline \multirow[t]{2}{*}{ Open reduction } & 23 \\
\hline & Number of patients \\
\hline Closed pinning and open reduction & 6 \\
\hline Bilateral closed pinning & 3 \\
\hline Bilateral open reduction & 1 \\
\hline
\end{tabular}

taken one year after open reduction showed diminution of joint space and rarefaction. The pins were removed early because it was feared that infection might be present, but culture showed them to be sterile, and examination of the blood showed no evidence of reaction to infection. One year later the condition of the joint was unchanged, but four years after operation there was some evidence of regeneration of articular cartilage (Figs. 20 and 21).

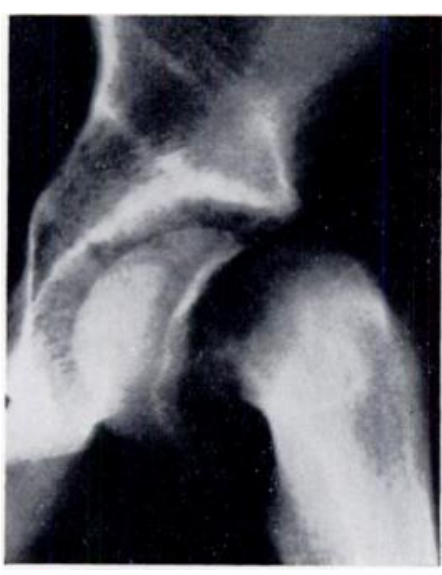

FIG. 18

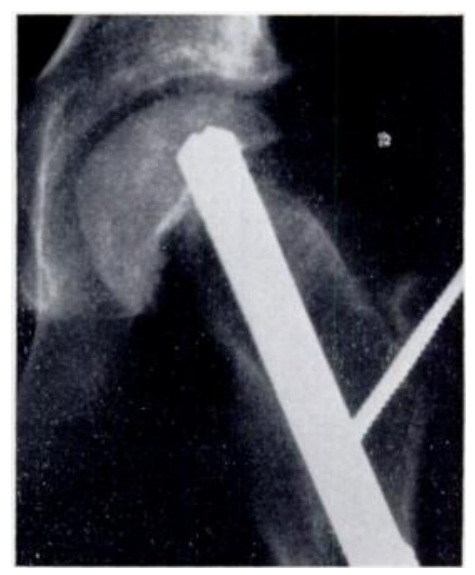

FIG. 19

Avascular necrosis of femoral head. Figure 18-Radiograph taken before reduction, showing severe degree of slip. Figure 19-Six weeks after operation: relative increase of density of femoral head.

At the age of twenty-one, and five years after operation, the patient felt only some stiffness of the hip after walking a few miles; he could swim and play a little football and could stand all day at work. The hip could be flexed from 0 to 90 degrees and had 10 degrees of abduction as compared with 35 degrees on the healthy side.

In the second case there was severe lateral rotation deformity before operation (Fig. 22). Reduction by manipulation under anaesthesia was attempted at another hospital. After open reduction an adduction deformity developed and there was evidence of necrosis of articular 
cartilage (Fig. 23). The deformity was corrected by osteotomy but later relapsed slightly, and there was some evidence of regeneration of articular cartilage. Three years after operation the patient had no pain in the hip; flexion from 0 to 70 degrees was possible but the limb could not be abducted beyond the neutral position (Fig. 24).

In both cases then, defects in technique may have contributed to the poor result. It seems clear that both patients will have further trouble with their hips.

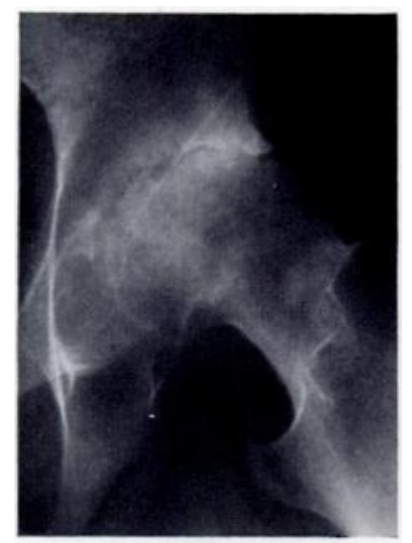

FIG. 20

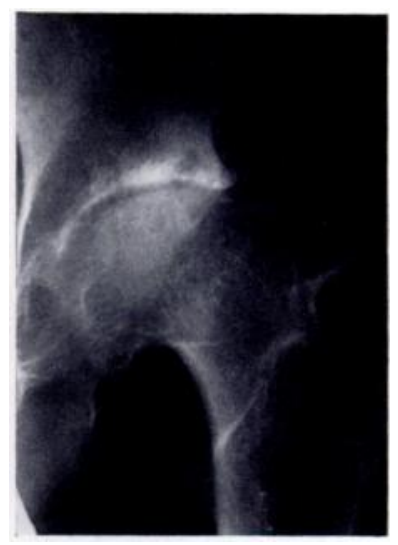

Fig. 21

Acute necrosis of cartilage. Figure 20-Radiograph taken two years after open reduction and pinning. Diminution of joint space and rarefaction of bones. Figure $21-$ Two years later: some evidence of regeneration of articular cartilage.

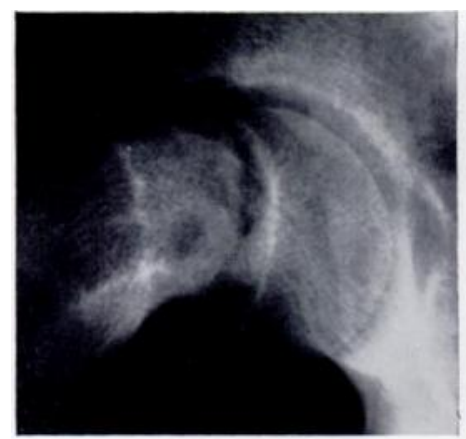

FIG. 22

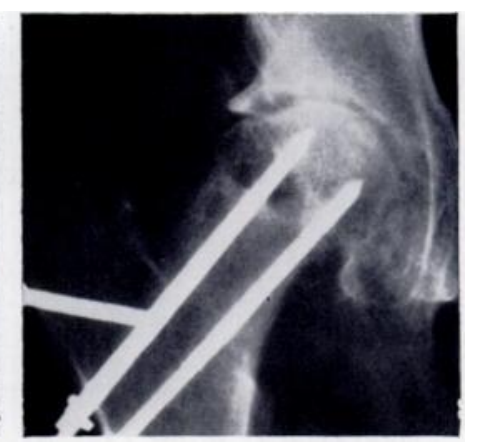

FIG. 23

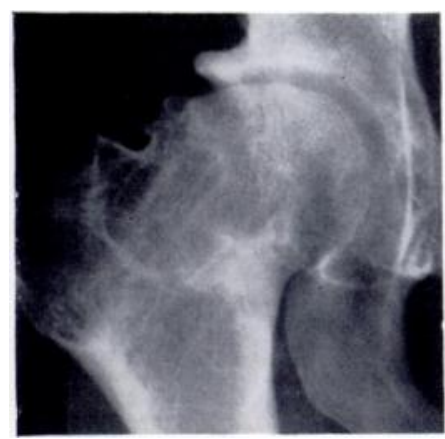

FIG. 24

Acute necrosis of cartilage. Figure 22-Radiograph showing condition before operation. Figure 23-Fifteen months after open reduction. Note the diminution of joint space. Figure 24-Three years after operation: some evidence of regeneration of cartilage.

\section{DISCUSSION}

It has been asked why one should do a difficult operation and one that might endanger the blood supply to the femoral head when an easier, and safer one, namely intertrochanteric osteotomy, is available. Both operations will correct the clinical deformity of the leg. but whereas the intertrochanteric operation leaves the anatomy of the hip joint grossly abnormal, cervical osteotomy makes it nearly normal again (Figs. 25 and 26). It is likely therefore that the later onset of degenerative arthritis is much more likely after the former operation. The point has not yet been proved, and until many patients treated by each method have been reviewed after at least twenty years it will remain undecided. However, Pearson and Riddell's (1964) study suggests that the late results of trochanteric osteotomy may be disappointing. 


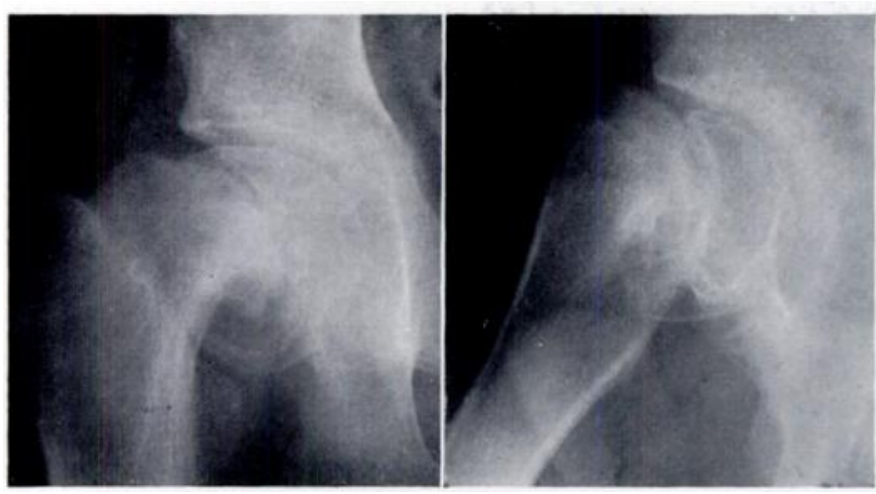

FIG. 25

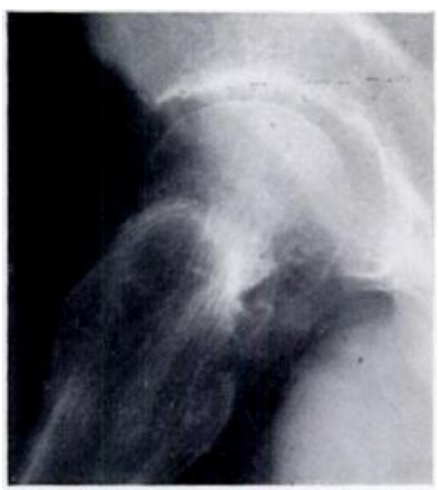

FIG. 26

A result of open reduction. Figure 25-Antero-posterior and (right) lateral radiographs showing condition before operation. Figure $2 \epsilon$ - Fifteen months after operation: excellent reposition and normally shaped head.

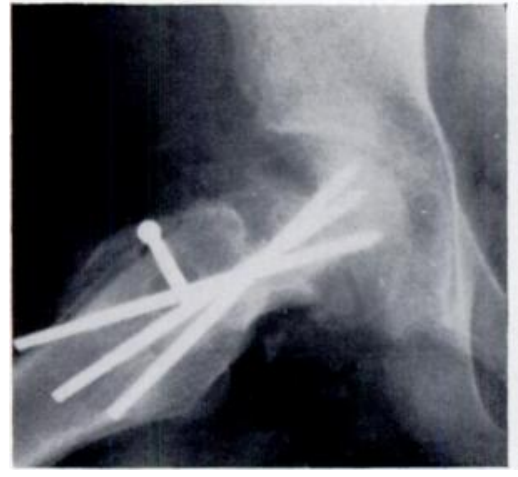

Fig. 27

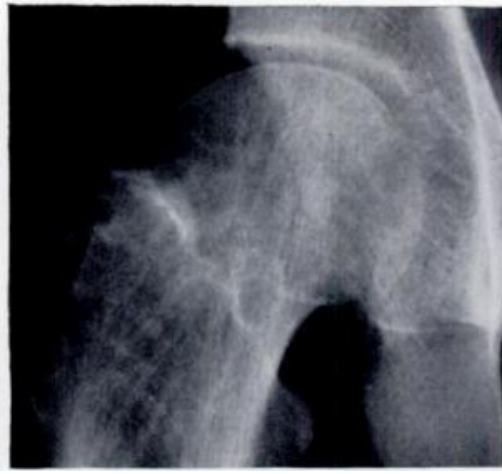

Fig. 28

A result of advancement of the synovium with its vessels. Figure 27-Radiograph taken two months after operation. Figure 28-One year later: normally shaped head and neck; no evidence of necrosis of head.

If cervical osteotomy is to be done, it should be done through a lateral approach with elevation of the trochanter so that a clear field of vision is obtained. The object should be to fix the head on the end of the neck without tension on the vessels. This is achieved either by freeing the vessels adequately or by adequate shortening of the femoral neck (Figs. 27 and 28).

I would like to thank $\mathrm{Mr} \mathrm{H}$. Osmond-Clarke for his continued interest and encouragement and $\mathrm{Mr}$ John Watts for providing the illustrations.

\section{REFERENCES}

MCFARL AND, B., and Osborne, G. (1954): Approach to the Hip. Journal of Bone and Joint Surgery, 36-B, 364. Pfarson, J. R., and Riddell, D. M. (1964): Subtrochanteric Osteotomy in the Treatment of Slipped Upper Femoral Epiphysis. Journal of Bone and Joint Surgery, 46-B, 155. 